\title{
Nanomechanics based investigation into interface thermomechanics of collagen and chitin based biomaterials
}

\author{
Qu, Tao; Tomar, Vikas, Purdue University, West LafayetteUnited States
}

\begin{abstract}
From the biological/chemical perspective, interface concepts related to cell surface/synthetic biomaterial interface and extracellular matrix/biomolecule interface have wide applications in medical and biological technology. Some findings regarding interfaces controlling biological reactions are like surfaces provide high accessibility for reaction, high surface area geometries that can be created to enhance reaction turnover rates, unique organic microenvironments that can enhance specific affinities and reactions, self-assembly in the plane of the interface that can be used to orient and space molecules with precision, and surface energy minimization can orient specific structures to interfaces. The focus of the presented work is on investigating thermal as well as mechanical properties of collagen-hydroxyapatite and chitin-calcite interfaces ubiquitous in nature. A new framework that predicts interface structure and hierarchy dependent thermal and mechanical properties of such interfaces is presented. Analyses point out the role of temperature, mechanical loading, and interface hierarchy in unique thermomechanical properties of biological interfaces. More importantly, interface topology and roughness are shown to play an important role in hierarchy dependent properties of biological materials. Based on this some biomimetic material design principles are analyzed.
\end{abstract}

